\title{
Visualization of magnetostructural transition in Heusler alloys by Magnetic Force Microscopy
}

\author{
Pavel Geydt ${ }^{1, *}$, Igor D. Rodionov ${ }^{2}$, Alexander B. Granovsky ${ }^{2}$, Ekaterina Soboleva ${ }^{1}$, Egor Fadeev ${ }^{1}$, Ivan $_{\text {S. Titov }}^{2,3}$, Igor \\ Dubenko $^{4}$, and Erkki Lähderanta ${ }^{1}$ \\ ${ }^{1}$ School of Engineering Science, Lappeenranta University of Technology (LUT), 53850 Lappeenranta, Finland \\ ${ }^{2}$ Faculty of Physics, Lomonosov Moscow State University (MSU), 119991 Moscow, Russia \\ ${ }^{3}$ Physics and Materials Science Research Unit, University of Luxembourg, 4365 Luxembourg, Luxembourg \\ ${ }^{4}$ Department of Physics, Southern Illinois University, Carbondale, IL 62901, USA
}

\begin{abstract}
Magnetostructural transition was observed in Ni-Mn-In-Cr Heusler alloy with help of Magnetic Force Microscopy (MFM). The crystal structure of a sample and characteristic temperatures of the phase transition were controlled by roentgenostructural phase analysis and magnetometry, respectively. It appeared prominently important to prepare the surface of the sample until the nanometer level of surface roughness. Magnetic study performed with scanning probe microscope revealed existence of magnetic domains, which were spread across the surface evenly. Further studies revealed that intensity of magnetic signal decreases as fading out of the contrast of the MFM images. It was found that location of domains shifted after the heating/cooling cycle above Curie temperature for the studied alloy. Location of new domain walls appeared correlating with surface scrapings and defects, whilst it became independent from those after heating until just $70^{\circ} \mathrm{C}$. The mechanism behind the observed transition is proposed.
\end{abstract}

\section{Introduction and Motivation}

Industrial and social needs are precursors of social and economic development, while scientific interest and creativity drive the state of technological advancement. Recently, sustainability paradigm requires more and more ecological, affordable and definitely non-toxic solutions for human scale development needs [1]. Latest three decades represented intensive growth of computerization and spreading of so-called smart technologies across human households. Unfortunately, even chemicals that are used inside coolers of typical refrigerators can hardly be considered as safe for life.

As a response to these challenges, one can observe a strong interest and investments oriented toward basic research of magnetic Heusler alloys. Heusler alloys represent a class of ternary intermetallic compounds where ferromagnetism emerges even in absence of 'typical' ferromagnetic materials [2] such as $\mathrm{Co}, \mathrm{Fe}, \mathrm{Ni}$ etc. Since the discovery of this phenomenon in 1903, NiMn-In became the most widely acclaimed type of Heusler alloy. Exactly Ni-Mn-In is considered as the most probable solution for having the magnetostructural phase transition near room temperature, while the transition is sharp and the difference in magnetization density between martensitic and austenitic phases is also significant. It was shown that addition of the fourth component can significantly affect properties of the alloy, including the characteristic temperatures [3]. Addition of chromium was considered since it is an antiferromagnetic material and can improve the magnetocaloric output and decrease the electrical resistivity of the material.

Typically, Heusler alloys are studied in bulk form by SQUID magnetometry [4], optical magnetic methods [5], calorimetry [5], volumetry [6] and standard structural approaches such as XRD [7]. However, neither of the abovementioned methods can reveal micro- and nanoscale details of a mechanism of magnetostructural transition. It is yet unclear how the austenitic phase emerges in elevated temperatures under magnetic field, while martensitic phase experience paramagnetic transition in equivalent temperatures. Magnetic memory effect, i.e. hysteresis of magnetization, inhomogeneity of chemical composition within the samples and dissimilarity between thermodynamic characteristics of the surface and bulk material veil the definitive mechanism of transformation, which is nowadays far from being well understood.

Scanning Probe Microscopy (SPM) is exactly an accurate and spatially local family of methods, but surprisingly, we noticed a strong deficit of works published with its results concerned with Heusler alloys. Thus, we decided to perform a Magnetic force Microscopy (MFM) study of Ni-Mn-In alloy under variable temperature with strive to answer on how the magnetostructural transition occurs within the sample.

\section{Sample preparation and Examination}

\footnotetext{
* Corresponding author: pavel.geydt@gmail.com
} 
Polycrystalline samples of $\mathrm{Ni}_{48} \mathrm{Mn}_{37} \mathrm{In}_{13} \mathrm{Cr}_{2}$ were fabricated with arc melting technique. Melting was repeated at least 7 times in argon atmosphere, followed by annealing of $\sim 3 \mathrm{~g}$ ingots in vacuum $10^{-4}$ torr under heating $\mathrm{T}=850^{\circ} \mathrm{C}$ and cooling in water. After cutting and grinding performed with water protection from extensive reheat, the sample was polished by abrasive paste containing $\sim 250 \mathrm{~nm}$ inclusions of diamond crystals on station EcoMet 250 (Buehler, USA). For details of the growth and polishing techniques, one can access [8].

Final verification of properties was acquired by energy-dispersive spectroscopy (EDS) and differential scanning calorimetry (DSC) (both not shown), and also X-ray diffraction (XRD), atomic force microscopy (AFM) and SQUID magnetometry. The roentgenostructural phase analysis (Fig. 1) have shown that both martensitic and austenitic phases are represented in the material at room temperature, which correlates well with previous studies of a similar system.

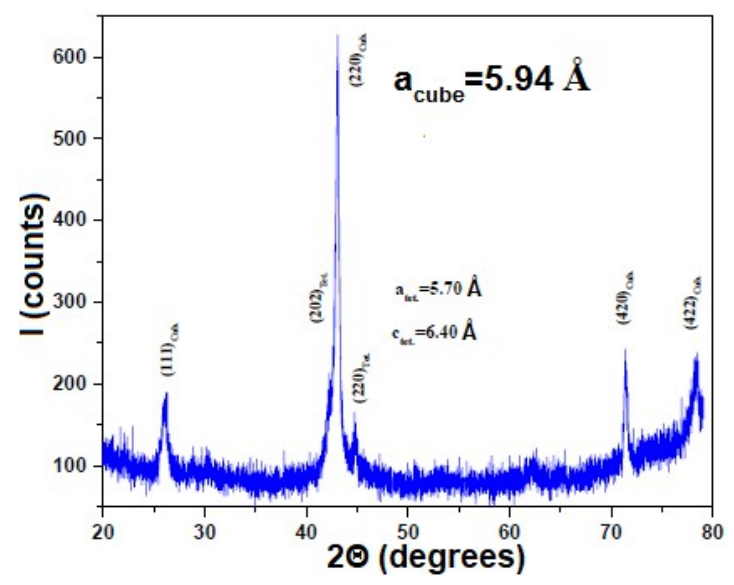

Fig. 1. XRD spectra for the Ni-Mn-In-Cr alloy.

Dependences of magnetization from temperature were studied with SQUID (Cryogenic R-S700X, UK). As it is visible from Figs. 2 and 3, the martensitic phase transition begins approximately at $320 \mathrm{~K}$. It was possible to observe the difference between the gram-weight sample and approximately ten-milligram piece of sample after polishing (Fig. 2). It was visible that the temperature of martensitic transition for a smaller sample was $\sim 10 \mathrm{~K}$ lower than for the initial one.

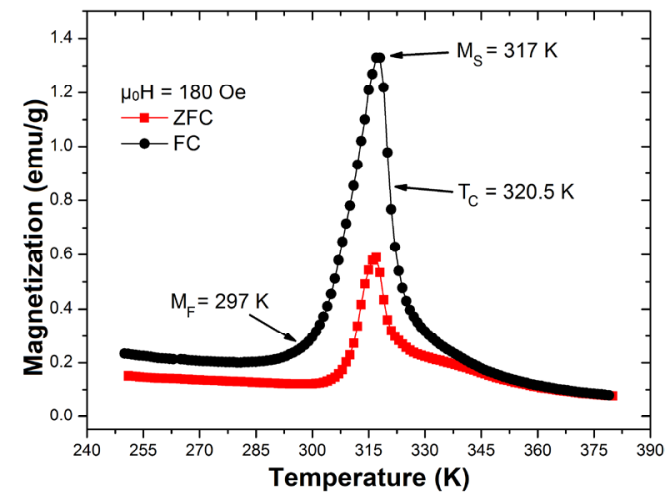

Fig. 2. Magnetization vs. temperature dependence for the Ni$\mathrm{Mn}$-In-Cr alloy under 180 Oe for milligram-weight sample.

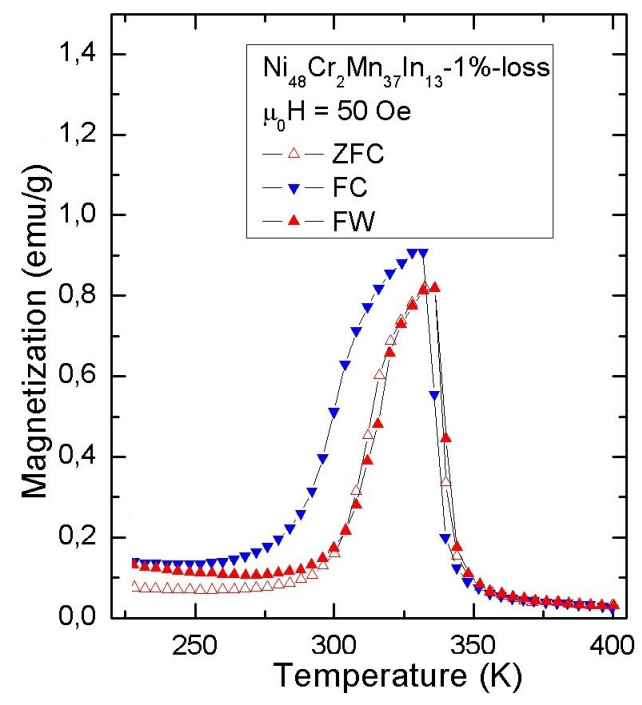

Fig. 3. Magnetization vs. temperature dependence for the Ni$\mathrm{Mn}-\mathrm{In}$-Cr alloy under $50 \mathrm{Oe}$ for gram-weight sample.

For the small sample, the entire material reaches the austenitic phase later than in Fig. 3, so that it appears that almost all austenitic phase is already paramagnetic.

Interesting accomplishments were attained from SPM study performed on Multimode 8 station (Bruker, USA), while they became possible solely due to the high-end polishing of the sample. In fact, more than two years and specific sample preparation were required to visualize the magnetic morphology with MFM. While previously samples could hardly be visualized (even to observe the topology), the polishing step permitted revealing separate magnetic domains within the smooth nearsurface layer of the alloy. Therefore, desirable roughness is justified in the level of few nanometers per 10x10 $\mu \mathrm{m}$ scan area.

\section{MFM during thermal cycling}

Magnetic domains were visualized on the flat surface of the sample of Ni-Mn-In-Cr Heusler alloy. Approximately $1 \mu \mathrm{m}$ wide, they were homogeneously spread on the entire surface, which was registered in $100 \times 100 \mu \mathrm{m}$ images taken in room temperature (partly represented in Fig. 4). Size of the domains was not limited by imaging resolution, because the tip radius of the microscopic probe MFMV (Bruker, USA) was $\sim 40$ $\mathrm{nm}$ and lift scan distance was $\sim 40 \mathrm{~nm}$.
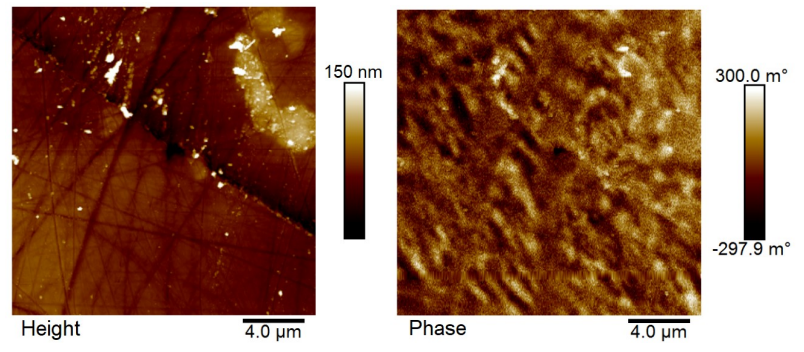

Fig. 4. Topology (Height) and Magnetic map (Phase) of the Ni-Mn-In-Cr sample for scan area 20x20 $\mu \mathrm{m}$. 
We need to admit that imaging of magnetic morphology with MFM is based on resonant oscillations of the probe during each scanning/tapping pattern. Typically, position of the resonance peak shifts to the lower values under heating, which is associated with decrease of elastic strength of the probe's material. Furthermore, intensity of resonant oscillations Arb decline, which is usually visible as decrease in height of the resonance peak. This effect is also associated with decline of stiffness of the probe and is to limited extent compensated by strengthening of the oscillations caused by decreased density of the heated ambient air. We observed that change of the peak followed the criterion $60 \mathrm{~Hz} / \mathrm{K}$ and $-1.5 \%$ Arb $/ \mathrm{K}$ (Fig. 5). All measurements were carried out in gas cell, containing room air. The scanned area was separated from atmosphere as a specialized Gas cell (Bruker, USA), where this chamber was heated by the preinstalled thermal application controller CLS200 (Watlow, USA).

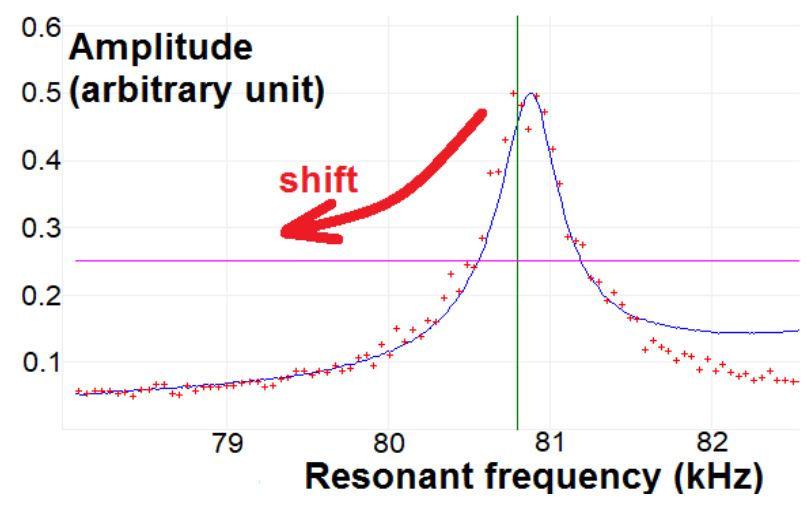

Fig. 5. Schematics of the change of resonance peak of SPM probe observed during thermal cycle.

Such control of the environment lead to accurate registration of the same $7 \times 7 \mu \mathrm{m}$ area of the sample, though thermal drift was compensated/controlled by enlarged scan sizes of all subsequent images. We performed the heating cycle with following temperatures 25-35-45-50-55-60-70-50-35 ${ }^{\circ} \mathrm{C}$, so that peculiar changes of the domain structures becomes clear from selective temperatures in Fig. 6. Comparing with the SQUID results, it was possible to highlight that surface of the sample experienced a phase transition $\sim 5^{\circ} \mathrm{C}$ lower than the bulk material, while such phenomenon was reported by another research group previously [9]. Furthermore, it was possible to register the change in position of the domains with heating (see consecutive temperatures in Fig. 6). We suggest that mechanical stresses during the grinding/polishing have persisted within the solid material and were concentrated in close proximity to the scrapings appeared from the diamond particles in polishing suspension. We assume that crystal structure also experienced change near these edges. Since these centers have captured additional tension energy, they tended to release it, which is associated with a lower temperature of phase transition observed previously by other groups and us. Domains vanished above the Curie temperature, while they appeared again when sample was cooled in external field of $\sim 180$ Oe.
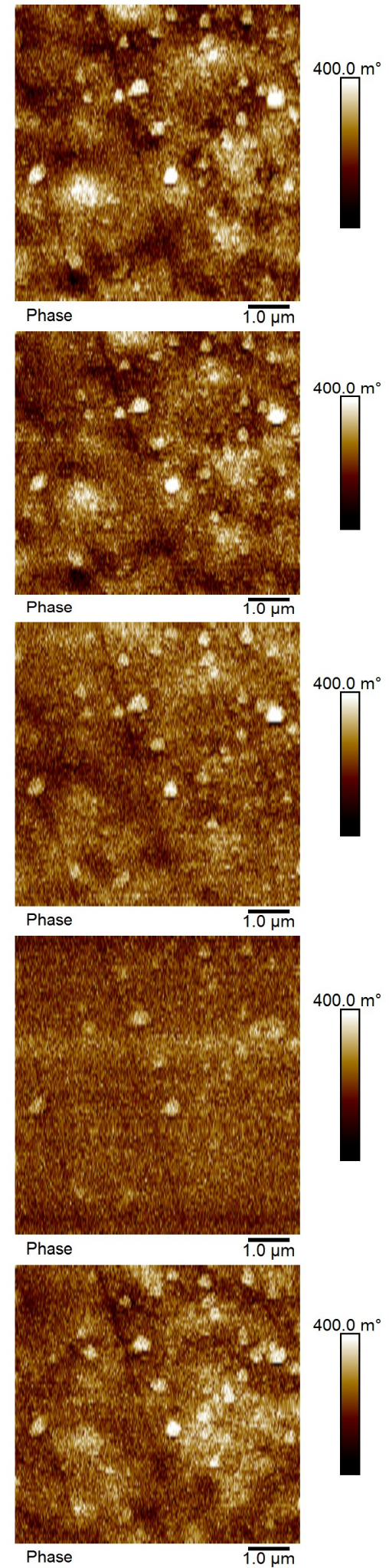

Fig. 6. Sequence of thermal MFM study represented as 25-3555-70-35 ${ }^{\circ} \mathrm{C}$. All MFM Phase images are presented for the same scanned area of $7 \times 7 \mu \mathrm{m}$.

Remarkably, the boundaries of studied magnetic domains emerged in new locations. Since these magnetic domains are expected to represent mixture of martensitic and austenitic phases, then we can highlight that exactly the magnetostructural transition has been visualized by MFM for the first time. 


\section{References}

1. D. Griggs, M. Stafford-Smith, O. Gaffney, J. Rockström et al., Nature 495 (2013)

2. A. Herdt, Exploring the electronic properties of novel spintronic materials by photoelectron spectroscopy (Dissertation, Jülich, 2012)

3. A. Zhukov, Novel Functional Magnetic Materials (Springer, 2016)

4. A. Quetz, Y. S. Koshkid'ko, I. Titov I., I. Rodionov et al., J. All. Comp. 683 (2016)

5. A. Novikov, A. Sokolov, E. A. Gan'shina, I. S. Dubenko, JMMM 432 (2017)

6. P. I. Polyakov, V. V. Slyusarev, V. V. Kokorin, S. M. Konoplyuk et al., J. Mater. Eng. Perform. 23 (2014)

7. S. Pandey, A. Quetz, A. Aryal, I. Dubenko et al., Magnetochemistry 3 (2017)

8. I. Titov, Influence of magnetism on the martensitic transition and related magnetocaloric effect in NiMn-based Heusler alloys (Dissertation, Duisburg-Essen, 2015)

9. K. Horakova, V. Chab, O. Heczko, V. Drchal et al., J. Appl. Phys 120 (2016) 